\title{
A Case of Acute Aortic Dissection Presenting Case A Case of Acute Aortic Dissection Presenting Report with Chest Pain Relieved by Sublingual Nitroglycerin
}

\author{
Hyun Joo Kim, Hyun-Ki Lee, Belong Cho*
}

Department of Family Medicine, Seoul National University Hospital, Seoul National University College of Medicine, Seoul, Korea

Acute aortic dissection is an uncommon disorder which can have fatal results in the event of treatment delay or misdiagnosis. This case examines a 77-year-old woman presenting with chest pain relieved by nitroglycerin. She was referred to the emergency room with clinical suspicion of acute coronary syndrome (ACS). However, she was later diagnosed with acute aortic dissection and an emergency operation was performed with successful recovery. Aortic dissection may manifest in various ways depending on the site involved and may mimic other disorders such as ACS or pulmonary embolism. Therefore, clinicians must always be aware of aortic dissection and its different clinical manifestations must be understood.

Keywords: Aortic Dissection; Acute Coronary Syndrome; Chest Pain; Diagnosis; Symptom

\section{INTRODUCTION}

Aortic dissection is a relatively uncommon, though catastrophic illness which requires early and accurate diagnosis and treatment for patient survival. The incidence of acute aortic dissection in the general population is estimated to range from 2.6 to 3.5 per 100,000 person-years. ${ }^{1)}$ Twenty percent of patients with aortic dissection die before reaching the hospital and 30\% die during hospital admission. ${ }^{2)}$ Because acute aortic dissection can result in fatal complications, the mortality rate increases at the rate of $1 \%$ to

Received: May 8, 2013, Accepted: October 21, 2013

${ }^{*}$ Corresponding Author: Belong Cho

Tel: +82-2-2072-2195, Fax: +82-2-766-3276

E-mail: belong@snu.ac.kr

Korean Journal of Family Medicine

Copyright (C) 2013 The Korean Academy of Family Medicine

(a) This is an open-access article distributed under the terms of the Creative Commons Attribution Non-Commercial License (http://creativecommons.org/licenses/by-nc/3.0) which permits unrestricted noncommercial use, distribution, and reproduction in any medium, provided the original work is properly cited.
$2 \%$ per hour without any treatment. ${ }^{3)}$ Therefore, fast and accurate diagnosis of aortic dissection is crucial and one must maintain a high index of suspicion.

However, the diagnosis of acute aortic dissection has many potential difficulties. Aortic dissection may mimic other more common conditions, such as coronary ischemia, pulmonary embolism, heart failure, stroke, and acute abdominal illness. ${ }^{4)}$ Differential diagnosis of aortic dissection to acute coronary syndrome (ACS) may be of particular importance because thrombolytic therapy may be fatal in aortic dissection.

Most common presenting symptoms of acute aortic dissection are acute pain in the chest or back. ${ }^{5,6)}$ Pain may be presented alone, or it can be presented with syncope, signs of congestive heart failure, or signs of cerebrovascular accident. ${ }^{7)}$ Painless aortic dissection is uncommon but is possible with only presenting symptoms and signs of congestive heart failure, cerebrovascular accident, and pulse loss. A study which evaluated factors contributing to delays in diagnosis, delays mostly occurred in female patients with atypical symptoms that were not abrupt or did not include chest, back, or any pain. ${ }^{8)}$ Therefore, atypical 
symptoms of aortic dissection should always be considered.

\section{CASE REPORT}

A 77-year-old Korean woman came to the outpatient clinic of the department of family medicine, Seoul National University, Seoul, Korea, presenting with chest pain that started the day before. She had a medical history of hypertension and had been on medication (losartan and aspirin) for 5 years. The day before, at about 7 PM she suddenly felt a strong chest pain of numerical rating scale (NRS) 8-10 in the substernal area. She described the character of the pain as oppressive without radiating pain. This excruciating pain lasted for about 10 minutes combined with a doomed feeling, diaphoresis, and difficulty in taking deep

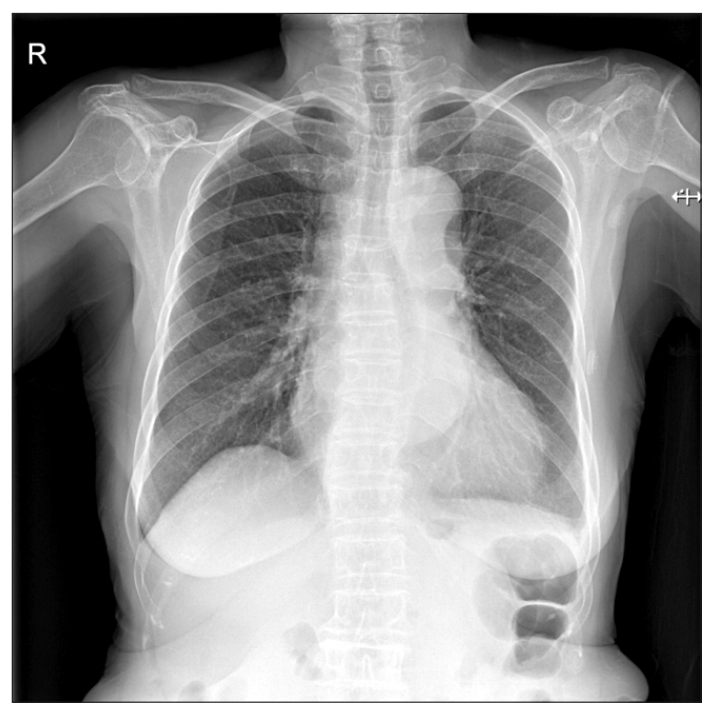

Figure 1. Chest radiograph: mild mediastinal widening with no active lung lesions. breaths. She also felt slight faintness with urinary incontinence. She visited a local clinic the next morning and chest pain was relieved to NRS 3-4 after 3 tablets of sublingual nitroglycerin. When she came to our outpatient clinic, she presented with moderate chest pain of $3 / 4$ on the NRS. She looked acutely ill, and nothing specific was detected in her heart and lung sounds. Under assessment of ACS she was immediately referred to the emergency department. Initial vital signs at the emergency room were stable: blood pressure $108 / 75 \mathrm{~mm} \mathrm{Hg}$, pulse rate $82 / \mathrm{min}$, respiration rate $20 / \mathrm{min}$, body temperature $36.6^{\circ} \mathrm{C}$, saturation 99\%. Electrocardiogram (EKG) was immediately taken which showed normal sinus rhythm with nonspecific ST deviation. Laboratory findings were as follows: creatine kinase (CK) 129 $\mathrm{IU} / \mathrm{L}, \mathrm{CK}$-myocardial band $3.0 \mathrm{IU} / \mathrm{L}$, troponin $\mathrm{I} 0.03 \mathrm{ng} / \mathrm{mL}$, C-reactive protein $0.59 \mathrm{mg} / \mathrm{dL}$, white blood cell $8,900 / \mu \mathrm{L}$, hemoglobin $11.4 \mathrm{~g} / \mathrm{dL}, \mathrm{D}$-dimer assay $13.60 \mathrm{ug} / \mathrm{mL}$. Cardiac enzyme levels were all within normal limits and nothing specific was found on the laboratory tests except an increase in D-dimer. The chest radiograph showed mild mediastinal widening with no active lung lesions (Figure 1). From the data above, it was less likely that the cause of chest pain was from ACS. Therefore, an additional imaging study was performed: computed tomography (CT) angiography + 3-dimensional aorta (contrast) (Figure 2). Surprisingly, the CT results showed aortic dissection type A starting from the distal ascending aorta with its distal extent just proximal to the right main renal artery (Figure 3). The diameter of the ascending aorta was $50 \mathrm{~mm}$ and there was no evidence of coronary artery os involvement. The thoracic surgery department was immediately contacted and the patient had an emergency operation: replacement of ascending aorta, proximal arch, and innominate artery with Hemashield graft and open heart
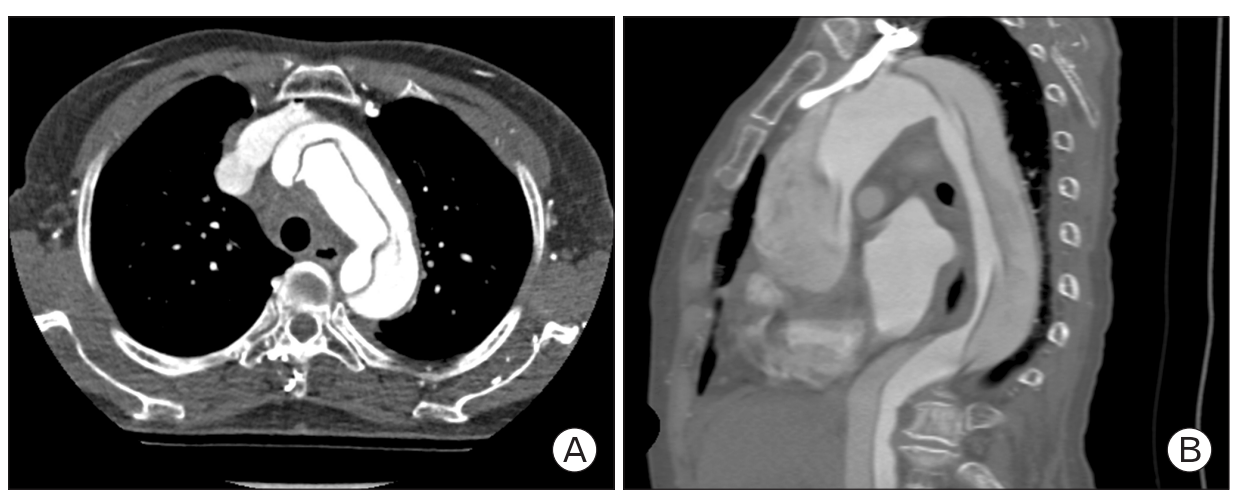

Figure 2. Computed tomography angio + 3-dimensional heart: aortic dissection type A starting from the distal ascending aorta. 


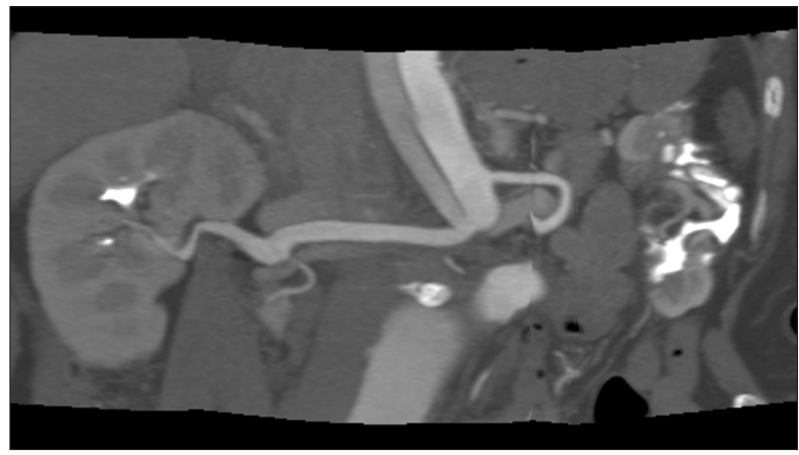

Figure 3. Computed tomography angio + 3-dimensional heart: distal extent of dissection just proximal to right main renal artery.

valvuloplasty of the aortic valve due to mild aortic regurgitation. The patient showed postoperative atrial fibrillation, which was managed by continuous infusion of amiodarone. Otherwise, she recovered well from the surgery and was discharged 18 days after the operation, with short-term follow-up at the outpatient clinic of thoracic surgery.

\section{DISCUSSION}

This case reports a 77-year old woman presenting with symptoms of chest pain that were thought to be from ACS, but later diagnosed as acute aortic dissection type A. According to Hagan et al., ${ }^{6}$ ) up to $30 \%$ of patients later found to have aortic dissection are initially suspected of having other conditions, such as ACSs, aneurysms, pericarditis, pulmonary embolism, aortic stenosis, or even cholecystitis. Despite recent advances in diagnostic methods, misdiagnosis occurs in $25 \%$ to $50 \%$ of patients on initial evaluation with symptoms mimicking those of acute myocardial infarction and other cardiovascular disorders. ${ }^{9,10)}$ Acute aortic syndrome is frequently confused with ACS, leading to delayed diagnosis and inappropriate treatment with antiplatelet, antithrombin, and fibrinolytic therapies. Exposure to these agents is associated with hemodynamic instability, hemorrhagic pleural and pericardial effusions, increased hemorrhagic postoperative complications, and a trend toward increased mortality. ${ }^{10)}$ Therefore not only is it important to have a clinical suspicion of aortic dissection in patients with abrupt chest pain, but also in patients with unexplained syncope, stroke, acute onset of congestive heart failure, or symptoms of acute ischemia of extremities or viscera.

First of all, it is important to assess risk factors of aortic dissection through thorough history taking. Aortic dissection occurs more often in men (1.55:1) and increases with age. ${ }^{11)}$ Doctors must ask about patient's underlying disease such as hypertension, atherosclerosis, or any preexisting aortic aneurysm. In this case report, the patient had a history of hypertension. Family history of aortic disease may also contribute as a risk factor. ${ }^{12)}$ Iatrogenic causes of aortic dissection include cardiac catheterization, angioplasty, or cardiac surgery. For younger patients, history of collagen diseases such as Marfan syndrome and Ehlers-Danlos syndrome, bicuspid aortic valve, aortic coarctation, and Turner syndrome should be investigated.

The most common presenting symptom of acute aortic dissection is chest pain which accounts for 79 percent of type A dissections and 63 percent of type $B$ dissections. ${ }^{6}$ The character of the pain in aortic dissection is typically sharp, tearing, and ripping with an abrupt onset. The severity of the pain is excruciating usually with maximum severity at the time of onset. Pain in acute aortic dissection may change its location with further extension of dissection. In contrast, the pain associated with acute ACS starts slowly, gains intensity with time, and its character is usually more oppressive and dull. ${ }^{7)}$ In this case, the patient presented with chest pain with abrupt onset, with its maximum severity at the time of onset. However, she described the character of the pain as oppressive rather than sharp or tearing. Although the character of the pain did not match the typical pain associated with aortic dissection, a physician must always suspect aortic dissection because atypical presentations can be possible and aortic dissection may be combined with ACS.

In addition, severity of chest pain in this case was relieved by sublingual nitroglycerin. Nitroglycerin acts by relaxing smooth muscle and dilating veins, arteries, and coronary arteries. Venodilation lowers preload and therefore reduces wall stress, resulting in a decrease in myocardial oxygen demand. Nitroglycerin could have temporally decreased chest pain by lowering the patient's blood pressure and reducing wall stress. However, as vasodilators may increase the force of left ventricular ejection, Nitroglycerin alone must be used with caution.

Uncommonly, painless dissection has been reported with an incidence of $6.4 \%$ to $17 \%{ }^{13,14)}$ Uncommon symptoms include syncope, congestive heart failure symptoms, cardiac tamponade, 
lower extremity weakness, paraplegia, mesenteric ischemia, or peripheral ischemia.

Physical examination can provide important clues to diagnose aortic dissection. Blood pressure measurement of right and left arm or legs must be done. High blood pressure is found in $70 \%$ of distal dissection and $35 \%$ of proximal dissection. ${ }^{6}$ Pulse check must be performed on bilateral upper and lower extremities as less than $20 \%$ of patients have pulse deficit or pulse difference resulting from intimal flap or compression by hematoma. ${ }^{6}$ ) Diastolic murmur indicative of aortic regurgitation is present in about half of the patients with proximal aortic dissection. Occasionally the murmur can be very faint, and it is probable that the physician did not recognize the murmur in this case. Neurologic examinations to evaluate neurological deficits such as loss of consciousness and ischemic paresis are important as they can occur in up to $40 \%$ of patients with proximal aortic dissection. $^{15)}$

Despite continuous research into novel biochemical diagnostic markers such as smooth muscle myosin heavy chain, there is still no specific blood test marker that can be used to diagnose aortic dissection. $\mathrm{D}$-dimer may be used as an exclusive method to rule out pulmonary embolism and aortic dissection if done within 24 hours after symptom onset. ${ }^{16)}$ An ECG must be performed on all patients for the differential diagnosis of acute myocardial infarction of aortic dissection. ${ }^{17)}$ However, clinicians must always keep in mind that aortic dissection can be combined with myocardial infarction if the dissecting membrane involves the coronary arteries. Thirty one percent of aortic dissection patients present with a normal ECG. Therefore ECG cannot be used as a diagnostic tool. ${ }^{5)}$ The role of chest radiographs remains unclear in the diagnosis of aortic dissection since 60-90\% show abnormal results such as abnormal aortic contour, mediastinal widening, or aortic shadow widening.

For definitive diagnosis, imaging such as CT, magnetic resonance imaging (MRI), transesophageal echocardiography (TTE), or aortography must be performed. With improvement of CT, CT is the most often used modality to diagnose aortic dissection because of its high specificity and sensitivity, and its availability. However CT has its limitations since it cannot detect aortic regurgitation. MRI also has high specificity and sensitivity, but the test is time consuming and less available. Therefore, MRI is usually used for follow-up imaging or for chronic dissections.
TEE is usually used for hemodynamically unstable patients since it can be performed at the bedside or in an operating room for emergency. ${ }^{7)}$

Considering the cost and radiation exposure, using imaging modalities on every patient suspected to have acute aortic syndromes is extremely costly and ineffective. Therefore, American Heart Association presented guidelines with a risk assessment tool which could be used to stratify patients from low risk to high risk. ${ }^{18)}$ Depending on the risk group this guideline provides clinicians a framework of additional diagnostic methods based on the probability of disease. Research performed in 2011 tested the sensitivity of the above guidelines which presented a high sensitivity of $95.7 \% .{ }^{18)}$ According to this risk score, the patient in this case has aortic dissection detection risk score of 1 (chest pain with abrupt onset), and according to this evaluation pathway, this patient will have undergone aortic imaging for evaluation.

This case report emphasizes the importance of clinical suspicion of aortic dissection and discusses the important clinical presentations of aortic dissection and its diagnostic methods. Furthermore, recent studies on aortic dissection detection risk scores have been discussed. Many patients will visit not only the emergency room, but the out-patient clinic, presenting symptoms of chest pain only, or any symptoms of aortic dissection. Clinicians must always be aware of aortic dissection even though the patient presents only with chest pain that might be similar to chest pain of ACS, and thorough history taking and physical examination must be performed.

\section{CONFLICT OF INTEREST}

No potential conflict of interest relevant to this article was reported.

\section{REFERENCES}

1. Clouse WD, Hallett JW Jr, Schaff HV, Spittell PC, Rowland CM, Ilstrup DM, et al. Acute aortic dissection: populationbased incidence compared with degenerative aortic aneurysm rupture. Mayo Clin Proc 2004;79:176-80.

2. Olsson C, Thelin S, Stahle E, Ekbom A, Granath F. Thoracic 
aortic aneurysm and dissection: increasing prevalence and improved outcomes reported in a nationwide populationbased study of more than 14,000 cases from 1987 to 2002 . Circulation 2006;114:2611-8.

3. Shirakabe A, Hata N, Yokoyama S, Shinada T, Suzuki Y, Kobayashi N, et al. Diagnostic score to differentiate acute aortic dissection in the emergency room. Circ J 2008;72:98690.

4. Braverman AC. Aortic dissection: prompt diagnosis and emergency treatment are critical. Cleve Clin J Med 2011;78: 685-96.

5. Spittell PC, Spittell JA Jr, Joyce JW, Tajik AJ, Edwards WD, Schaff $\mathrm{HV}$, et al. Clinical features and differential diagnosis of aortic dissection: experience with 236 cases (1980 through 1990). Mayo Clin Proc 1993;68:642-51.

6. Hagan PG, Nienaber CA, Isselbacher EM, Bruckman D, Karavite DJ, Russman PL, et al. The International Registry of Acute Aortic Dissection (IRAD): new insights into an old disease. JAMA 2000;283:897-903.

7. Erbel R, Alfonso F, Boileau C, Dirsch O, Eber B, Haverich A, et al. Diagnosis and management of aortic dissection. Eur Heart J 2001;22:1642-81.

8. Harris KM, Strauss CE, Eagle KA, Hirsch AT, Isselbacher EM, Tsai TT, et al. Correlates of delayed recognition and treatment of acute type A aortic dissection: the International Registry of Acute Aortic Dissection (IRAD). Circulation 2011;124:1911-8.

9. Klompas M. Does this patient have an acute thoracic aortic dissection? JAMA 2002;287:2262-72.

10. Hansen MS, Nogareda GJ, Hutchison SJ. Frequency of and inappropriate treatment of misdiagnosis of acute aortic dissection. Am J Cardiol 2007;99:852-6.

11. Meszaros I, Morocz J, Szlavi J, Schmidt J, Tornoci L, Nagy L, et al. Epidemiology and clinicopathology of aortic dissection. Chest 2000;117:1271-8.

12. Hasham SN, Lewin MR, Tran VT, Pannu H, Muilenburg A, Willing M, et al. Nonsyndromic genetic predisposition to aortic dissection: a newly recognized, diagnosable, and preventable occurrence in families. Ann Emerg Med 2004;43:79-82.

13. Park SW, Hutchison S, Mehta RH, Isselbacher EM, Cooper JV, Fang J, et al. Association of painless acute aortic dissection with increased mortality. Mayo Clin Proc 2004;79:1252-7.

14. Imamura H, Sekiguchi Y, Iwashita T, Dohgomori H, Mochizuki K, Aizawa K, et al. Painless acute aortic dissection: diagnostic, prognostic and clinical implications. Circ J 2011;75:59-66.

15. Fann JI, Sarris GE, Mitchell RS, Shumway NE, Stinson EB, Oyer PE, et al. Treatment of patients with aortic dissection presenting with peripheral vascular complications. Ann Surg 1990;212:705-13.

16. Hugli OW. Letter by Hugli regarding article, "Diagnosis of acute aortic dissection by $\mathrm{D}$-dimer: the International Registry of Acute Aortic Dissection Substudy on Biomarkers (IRADBio) experience”. Circulation 2010;121:e23.

17. Kamp TJ, Goldschmidt-Clermont PJ, Brinker JA, Resar JR. Myocardial infarction, aortic dissection, and thrombolytic therapy. Am Heart J 1994;128(6 Pt 1):1234-7.

18. Rogers AM, Hermann LK, Booher AM, Nienaber CA, Williams DM, Kazerooni EA, et al. Sensitivity of the aortic dissection detection risk score, a novel guideline-based tool for identification of acute aortic dissection at initial presentation: results from the international registry of acute aortic dissection. Circulation 2011;123:2213-8. 